\title{
How many quasiplatonic surfaces?
}

\author{
Jan-Christoph Schlage-Puchta, Jürgen Wolfart \\ Mathematisches Institut der Universität, Eckerst. 1, D-79104 Freiburg \\ Mathematisches Seminar der Universität, Postfach 1119 23, D-60054 Frankfurt a.M. \\ jcp@math.uni-freiburg.de wolfart@math.uni-frankfurt.de
}

\begin{abstract}
We show that the number of isomorphism classes of quasiplatonic Riemann surfaces of genus $\leq g$ has a growth of type $g^{\log g}$. The number of non-isomorphic regular dessins of genus $\leq g$ has the same growth type.
\end{abstract}

MSC Index: 20E07, 30F10, 14H30.

Keywords: Dessin d'enfants, compact Riemann surfaces, subgroup growth.

Quasiplatonic Riemann surfaces $X$ of genus $g>1$ can be characterized in many different ways (see e.g. [Wo2, Thm. 4], or [Si2]), e.g. by the property that the orders of their automorphism groups are isolated local maxima in the corresponding moduli space of Riemann surfaces of genus $g$. For the present paper we will only use the equivalent statement that their universal covering groups $\Gamma$ are torsion free normal subgroups of finite index in some Fuchsian triangle groups $\Delta=\Delta(p, q, r)$ of signatures $(p, q, r)$, and that conversely the quotient $\Gamma \backslash \mathbf{H}$ of the upper half plane $\mathbf{H}$ by any finite index torsion free normal subgroup $\Gamma$ of a Fuchsian triangle group is a quasiplatonic surface $X$. The dessin it carries can be described as the quotient by $\Gamma$ of a $\Delta$-invariant tesselation of $\mathbf{H}$, with $\Delta / \Gamma$ acting as a group of automorphisms on $X$ and on the dessin.

Notations: Let $R(g ; p, q, r)$ be the number of normal torsion free subgroups $\Gamma$ of genus $g$ in $\Delta=\Delta(p, q, r)$, and let

$$
R(g):=\sum_{p, q, r} R(g ; p, q, r), \quad S(g):=\sum_{1<\gamma \leq g} R(\gamma)
$$

be the number of all non-isomorphic regular dessins of this genus $g$ and its summatory function. We will show first

Theorem 1 There are constants $g_{0}, c_{1}, c_{2}>0$ such that for all genera $g>g_{0}$

$$
g^{c_{1} \log g}<S(g)<g^{c_{2} \log g} .
$$


We will use the following result of T. Müller and the first named author (a simplified version of [MSP, Theorem 1]). For a group $\Gamma$, denote by $s_{n}^{\triangleleft}(\Gamma)$ the number of normal subgroups of index $n$ in $\Gamma$.

Lemma 1 Let $\Gamma$ be a finitely generated group, possessing a normal subgroup $N$ of finite index which maps surjectively onto a non-abelian free group. Then we have for $n>n_{0}$ the estimate $\sum_{\nu \leq n} s_{\nu}^{\triangleleft}(\Gamma) \geq n^{c \log n}$, where $n_{0}$ and c are positive constants depending on $\Gamma$.

Every Fuchsian triangle group has a torsion free normal subgroup of finite index, which is necessarily a surface group with at least 4 generators. Since an orientable surface group with $2 d$ generators maps surjectively onto a free group with $d$ generators, Lemma 1 can be applied to all Fuchsian triangle groups.

We now turn to the proof of Theorem 1. To obtain the lower bound, take three different primes $p, q, r$ giving a Fuchsian triangle group $\Delta=\Delta(p, q, r)$ with presentation

$$
\Delta=\left\langle\gamma_{0}, \gamma_{1} \mid \gamma_{0}^{p}=\gamma_{1}^{q}=\left(\gamma_{0} \gamma_{1}\right)^{r}=1\right\rangle .
$$

All normal subgroups of index $n>1$ are torsion free. By the Riemann-Hurwitz formula, their genus $g$ is related to $n$ via

$$
84(g-1) \geq n=(2 g-2)\left(1-\frac{1}{p}-\frac{1}{q}-\frac{1}{r}\right)^{-1}>2 g-2,
$$

and by Lemma 1 we have a lower bound for the summatory growth function

$$
\sum_{1<2(\gamma-1)<n} R(\gamma ; p, q, r) \geq|\{\Gamma \triangleleft \Delta(p, q, r) \mid 1<(\Delta(p, q, r): \Gamma) \leq n\}|>n^{c_{1} \log n}
$$

for all $n>n_{0}$ for some $n_{0}$ depending on $p, q, r$. Taking only that term in the sum $R(\gamma)=\sum_{p, q, r} R(\gamma ; p, q, r)$ coming from the triangle group $\Delta=\Delta(p, q, r)$ for the chosen prime triple signature we obtain

$$
\begin{gathered}
S(g) \geq \sum_{1<\gamma \leq g} R(\gamma ; p, q, r) \geq|\{\Gamma \triangleleft \Delta \mid 1<(\Delta: \Gamma) \leq 2(g-1)\}| \\
\geq|\{\Gamma \triangleleft \Delta \mid 1<(\Delta: \Gamma) \leq g\}|>g^{c_{1} \log g}
\end{gathered}
$$

for all $g \geq n_{0}$.

To prove the upper bound, recall Lubotzky's estimate $\nu^{6(\Omega(\nu)+1)}$ for the number of index $\nu$ normal subgroups in the free group with two generators ([LS, Thm. 2.7]) where $\Omega(\nu)$ denotes the number of prime divisors of $\nu$ counted with multiplicities. For any fixed Fuchsian triangle group $\Delta=\Delta(p, q, r)$ it implies

$$
|\{\Gamma \triangleleft \Delta \mid(\Delta: \Gamma) \leq n\}| \leq \sum_{\nu \leq n} \nu^{6(\Omega(\nu)+1)}<n^{c_{3} \log n}
$$


for some constant $c_{3}$, since $\Omega(\nu) \leq \log _{2} \nu$. This upper bound is a fortiori valid for the torsion free normal subgroups, hence

$$
\sum_{1<\gamma \leq g} R(\gamma ; p, q, r)<(84 g)^{c_{3} \log (84 g)}
$$

Since $\Delta / \Gamma$ has generators of orders $p, q, r$, we have moreover $R(\gamma ; p, q, r)=0$ for $p, q$ or $r>84 g(>|\Delta / \Gamma|)$, therefore

$$
S(g)=\sum_{1<\gamma \leq g} R(\gamma)<\sum_{p, q, r \leq 84 g}(84 g)^{c_{3} \log (84 g)}<(84 g)^{3+c_{3} \log (84 g)}<g^{c_{2} \log g}
$$

for all $g>g_{0}$ with suitable $c_{2}$ and $g_{0}$.

Theorem 2 Let $Q(g)$ denote the number of isomorphism classes of quasiplatonic Riemann surfaces of genera $\gamma, 1<\gamma \leq g$. With the same constants $g_{0}, c_{1}, c_{2}>0$ as in Theorem 1 we have for all $g>g_{0}$

$$
\frac{1}{120} g^{c_{1} \log g}<Q(g)<g^{c_{2} \log g}
$$

Proof. Since every quasiplatonic surface is obtained from a regular dessin (equivalently, from a torsion free normal subgroup in a triangle group), and is uniquely determined by that dessin, the upper bound follows from Theorem 1. The lower bound follows similarly, but a quasiplatonic surface can be obtained by up to five different types of regular dessins ([Gi]), and another overcount can happen: in a fixed triangle group $\Delta=\Delta(p, q, r)$ several torsion free normal subgroups can be $\mathrm{PSL}_{2}(\mathbf{R})$-conjugate, leading to isomorphic surfaces. In $[\mathrm{GW}]$, Thms. 5, 6, 7, it is shown that such conjugations take place in a finite extension of $\Delta$ which is again a triangle group. By Singerman's work [Si1] the maximal possible index between Fuchsian triangle groups is known to be 24 (occurring for $\Delta(2,3,7) \supset \Delta(7,7,7)$ ), so we have at most 120 normal subgroups counted in the proof of Theorem 1 leading to isomorphic surfaces (by a more detailed analysis, this number can considerably decreased). This gives the lower bound for $Q(g)$.

Another consequence of Thm. 1 is

Theorem 3 With the same constants $g_{0}, c_{1}, c_{2}>0$ as in Theorem 1, the number of nonisomorphic regular dessins in genus $g>g_{0}$ is $R(g)<g^{c_{2} \log g}$. Infinitely often we have

$$
g^{-1+c_{1} \log g}<R(g) \text {. }
$$

An analogous statement holds for the number of quasiplatonic surfaces of genus $g$. 
Remarks. 1) From the tables in [Wo1, Sec. 6] of regular dessins in genera $g \leq 4$ and work of Kuribayashi and Kimura [KK] for $g=5$ one may deduce

$$
S(5)=104 \quad \text { and } \quad Q(5)=37
$$

to be compared with $5^{\log 5} \approx 13$. [GAP] calculations [Sc] indicate that also for $5<g \leq 10$ one has always

$$
g^{\log g}<S(g)<g^{2 \log g} .
$$

2) Counting regular dessins in genera 0 and 1 is different from higher genera. In genus 0 the Riemann sphere is the only surface, however having an infinity of regular dessins defined by the cyclic and dihedral triangle groups of signatures $(1, n, n),(2,2, n)$ and those corresponding to the platonic bodies, i.e. $(2,3,3),(2,3,4),(2,3,5)$.

In genus 1 the triangle groups of signatures $(3,3,3),(2,3,6),(2,4,4)$ have infinitely many torsion free normal subgroups of finite index, acting by translations on the complex plane. As quotients one obtains infinitely many non-isomorphic elliptic curves with regular dessins, but all fall in two isogeny classes only (see [SiS]) having complex multiplication by the fields of third or forth roots of unity, respectively.

3) The most famous quasiplatonic surfaces are the Hurwitz curves whose automorphism groups attain the (according to Hurwitz) maximal possible order $84(g-1)$. Their universal covering groups are the torsion free normal subgroups of finite index in $\Delta(2,3,7)$. By the same or even easier arguments as above one can deduce that the number of non-isomorphic Hurwitz curves of genera $\gamma \leq g$ lies between $g^{c_{4} \log g}$ and $g^{c_{5} \log g}$ for all $g \geq g_{1}$ with suitable constants $g_{1}, c_{4}, c_{5}$.

Acknowledgement. The authors thank the referee for several useful remarks.

\section{References}

[GAP] The GAP Group: GAP — Groups, Algorithms, and Programming, http://www.gap-system.org

[Gi] E. Girondo: Multiply quasiplatonic Riemann surfaces, Experimental Mathematics 12 (4) (2003), 463-475

[GW] E. Girondo, J. Wolfart: Conjugators of Fuchsian groups and quasiplatonic surfaces, preprint 2004, http://www.math.uni-frankfurt.de/ wolfart, to appear in Quarterly J. Math. Oxford

[KK] A. Kuribayashi, H. Kimura: Automorphism Groups of Compact Riemann Surfaces of Genus Five, Journal of Algebra 134 (1990), 80-103 
[LS] A. Lubotzky, D. Segal: Subgroup growth, PM 212, Birkhäuser 2003

[MSP] T.W. Müller, J.-Chr. Schlage-Puchta: Normal growth of large groups, II, to appear in Archiv der Mathematik

[Sc] M. Schmitt: Reguläre Hypermaps, Diplomarbeit, Frankfurt 2001

[Si1] D. Singerman: Finitely Maximal Fuchsian Groups, J. London Math. Soc. (2) 6 (1972), 29-38

[Si2] D. Singerman: Riemann Surfaces, Belyi Functions and Hypermaps, pp. 43-68 in Topics on Riemann Surfaces and Fuchsian Groups (ed: E. Bujalance, A.F. Costa, E. Martinez), London Math. Soc. LNS 287, Cambridge UP 2001

[SiS] D. Singerman, R.I. Syddall: Belyi Uniformization of Elliptic Curves, Bull. London Math. Soc. 139 (1997), 443-451

[Wo1] J. Wolfart: Triangle groups and Jacobians of CM type, Frankfurt 2000, http://ww. math.uni-frankfurt.de/ wolfart

[Wo2] J. Wolfart: ABC for polynomials, dessins d'enfants, and uniformization - a survey, to appear in the proceedings of the ELAZ conference 2004, ed.: W. Schwarz, J. Steuding, http://www. math.uni-frankfurt.de/ wolfart 